\title{
Cinética de fermentação ruminal in vitro de alguns co-produtos gerados na cadeia produtiva do biodiesel pela técnica de produção de gás
}

\section{In vitro rumen fermentation kinetics of some co-products generated in the biodiesel production chain by gas production technique}

\author{
Ivone Yurika Mizubuti ${ }^{1 *}$; Edson Luis de Azambuja Ribeiro'; Elzânia Sales \\ Pereira $^{2,1}$; Andrea Pereira Pinto ${ }^{2}$; André Luiz Custódio Franco ${ }^{3}$; Mirna Adriane \\ Syperreck ${ }^{4}$; João Ricardo Rebouças Dórea ${ }^{5}$; Gianne Evans Cunha ${ }^{6}$; Matheus \\ Gabriel Maidana Capelari ${ }^{7}$; Elaine Barbosa Muniz ${ }^{8}$ \\ Resumo
}

\begin{abstract}
Objetivou-se com este experimento avaliar a cinética de fermentação ruminal de diferentes co-produtos gerados na cadeia produtiva do biodiesel, mediante o uso da técnica de semi-automática de produção de gases in vitro, usando um delineamento inteiramente casualizado, com cinco tratamentos e quatro repetições por tratamento. Os tratamentos foram: farelo de crambe; farelo de algodão; torta de crambe; torta de soja e torta de girassol. A torta de soja apresentou maior taxa de degradação de carboidratos não fibrosos (CNF), quando comparada aos demais alimentos, resultando em maior volume final de gases. $\mathrm{O}$ farelo de algodão e o farelo de crambe apresentaram taxa de degradação de CNF e produção de gases similares. Apesar da torta de crambe não diferir do farelo de crambe quanto a produção de gás na degradação de $\mathrm{CNF}$, houve diferença na taxa de degradação, sendo o maior valor verificado para a torta. O maior tempo de colonização (Lag time) foi requerido para a torta de soja e o menor para a torta de girassol. As maiores taxas de degradação de carboidratos fibrosos (CF) foram observadas na torta de soja e torta de crambe, e menor taxa na torta de girassol. A maior produção de gás oriunda da degradação de CF foi obtida para o farelo de crambe. A torta de soja e a torta de crambe foram os co-produtos com melhor perfil na cinética de fermentação ruminal no que diz respeito à degradação de carboidratos não fibrosos e carboidratos fibrosos. Palavras-chave: Alimentos alternativos, bicombustível, resíduo, taxa de degradação
\end{abstract}

\begin{abstract}
Fermentation kinetics rates of co-products generated in the supply chain of biodiesel were evaluated by in vitro gas production technique. Crambe meal, cottonseed meal, crambe cake, soybean cake and sunflower cake were evaluated. The soybean cake had higher rates of degradation of non-fiber carbohydrates (NFC), compared to other foods, resulting in higher volume of gas. Cottonseed meal and crambe meal had degradation rates of NFC and gas production similar. Although the crambe cake did not differ from crambe meal on gas production in the degradation of NFC, there were differences in the rate of degradation, being the highest value found for the cake. The biggest lag time was required
\end{abstract}

\footnotetext{
1 Profs. Drs. do Dept ${ }^{\circ}$ de Zootecnia. Universidade Estadual de Londrina, UEL. Pesquisadores do CNPq. E-mail: mizubuti@uel.br; elar@uel.br

2 Profs. Drs. do Dept ${ }^{\circ}$ de Zootecnia. Universidade Federal do Ceará, UFC. ${ }^{2,1}$ Pesquisadora do CNPq. E-mail: elzania@hotmail. com; deiapp@hotmail.com

3 Mestrando em Bioenergia. UEL, Londrina, PR. E-mail: andrefranco_agro@hotmail.com

4 Mestranda em Ciência Animal. UEL, Londrina, PR. E-mail: msyperreck@hotmail.com

5 Doutorando em Ciência Animal e Pastagem. Universidade de São Paulo, USP. E-mail: joaodorea@hotmail.com

6 Doutoranda em Ciência Animal. UEL, Londrina, PR. E-mail: gianneevans@hotmail.com

7 Graduando em Medicina Veterinária. UEL, Londrina, PR. Bolsista IC/CNPq. E-mail: matheus_capela@hotmail.com

8 Prof $^{\mathrm{a}}$. Dr ${ }^{\mathrm{a}}$. do Dept ${ }^{\mathrm{o}}$ de Zootecnia. Universidade Estadual do Oeste do Paraná, UNIOESTE. E-mail: ebmuniz@yahoo.com.br

* Autor para correspondência
} 
for soybean cake and the lowest for the sunflower cake. The highest rates of degradation of fibrous carbohydrates (FC) were observed in soybean cake and crambe cake, and lowest in sunflower cake. The highest gas production coming from the degradation of FC was obtained for the crambe meal. The soybean cake and crambe cake were the co-products with a better profile on rumen fermentation kinetics in relation to the degradation of non-fiber carbohydrates and fibrous carbohydrates.

Key words: Alternative food, biofuel, degradation rate, residue

\section{Introdução}

O Brasil está entre os maiores produtores e consumidores de biodiesel do mundo, com uma produção anual, em 2009, de 1,6 bilhões de litros e uma capacidade instalada, em janeiro de 2010, para cerca de 4,7 bilhões de litros, segundo a Agência Nacional de Petróleo, Gás Natural e Biocombustíveis (ANP, 2010). A agência estimou ainda que a mistura de $5 \%$ de biodiesel ao óleo diesel consumido no país, trará uma economia da ordem de US\$ 1,4 bilhão por ano devido à queda das importações de diesel e reduzirá em 3\% a emissão de dióxido de carbono $\left(\mathrm{CO}_{2}\right)$ em decorrência da queima do combustível no Brasil.

Quando a mistura obrigatória de biodiesel ao diesel era de apenas 3\%, o Brasil apresentava um potencial de produção de tortas e/ou farelos na ordem de $14.746 \mathrm{~kg} / \mathrm{ha} /$ ano (ABDALLA et al., 2008). Entre os co-produtos gerados pela diversidade de materiais graxos utilizados na cadeia do biodiesel, os farelos de algodão e de crambe, e as tortas de soja, de girassol e de crambe podem apresentar características desejáveis a um concentrado protéico de boa qualidade para alimentação de ruminantes. O domínio da tecnologia de utilização dos diversos tipos de co-produtos do biodiesel na alimentação animal ainda é escasso dependendo do co-produto, bem como, o conhecimento do mesmo pode maximizar a produtividade pecuária e agregar valores ao processo produtivo do biodiesel.

No sistema de produção animal, a alimentação representa o item de maior custo, sendo que está diretamente relacionado com a oferta de alimento de determinada região, tornando-se, portanto, fator determinante para a viabilização do sistema produtivo. Assim, faz-se necessário para o setor, a minimização de custos por meio da busca de alternativas à substituição de itens da dieta que possam melhorar as margens de lucro da atividade, apresentando desempenho pelo menos semelhantes ‘aqueles obtidos com itens de uso já consagrado (CNA, 2010).

Neste cenário, os co-produtos originados na cadeia produtiva do biodiesel no Brasil têm sido estudados como possíveis ingredientes de dietas para ruminantes. Estudos e técnicas que caracterizem o metabolismo ruminal destes coprodutos, como a técnica de produção de gases in vitro, é necessária para identificação de potenciais co-produtos passiveis de serem utilizados com eficiência na dieta de ruminantes em substituição a ingredientes convencionais.

O objetivo deste trabalho foi avaliar alguns co-produtos da cadeia produtiva do biodiesel com potencial de utilização na alimentação animal por meio dos parâmetros de fermentação ruminal in vitro utilizando a técnica de produção de gás.

\section{Material e Métodos}

O experimento foi conduzido no Laboratório de Analise de Alimentos e Nutrição Animal do Departamento de Zootecnia da Universidade Estadual de Londrina, localizado em Londrina-PR.

Os alimentos avaliados compreenderam diferentes co-produtos oriundos da cadeia produtiva do biodiesel, os quais constituíram os cinco tratamentos: farelo de crambe; farelo de algodão; torta de crambe; torta de soja e torta de girassol. Cada tratamento foi conduzido em quatro repetições.

Os co-produtos foram analisados para 
determinação dos teores de matéria seca (MS), matéria mineral (MM), matéria orgânica (MO), proteína bruta $(\mathrm{PB})$, extrato etéreo $(\mathrm{EE})$, fibra em detergente neutro (FDN), fibra em detergente ácido (FDA), lignina (LIG), proteína insolúvel em detergente neutro (PIDN) e ácido (PIDA), pelo método do acido sulfúrico, conforme metodologias descritas por Mizubuti et al. (2009). Os teores de carboidratos foram estimados conforme Sniffen et al. (1992); onde: carboidratos totais $(C T)=100$ $(\% \mathrm{~PB}+\% \mathrm{EE}+\% \mathrm{MM})$ e carboidratos não fibrosos $(\mathrm{CNF})=100-(\% \mathrm{FDNcp}+\% \mathrm{~PB}+\% \mathrm{EE}+\%$ cinzas $)$, em que, FDNcp equivale a parede celular corrigida para cinzas e proteína.

Para a estimativa dos valores de nutrientes digestíveis totais (NDT) (Tabela 1), procederam-se cálculos de acordo com a seguinte fórmula (NRC, 2001): NDT $=$ CNF-d + PB-d + (AG-d x 2,25) + FDNn-d - 7; em que o valor 7 refere-se ao NDT metabólico fecal, ou seja, a correção utilizada, uma vez que as frações digestíveis dos alimentos consideradas para o cálculo do NDT referem-se à digestibilidade verdadeira e não aparente; CNF-d equivale a carboidratos não fibrosos digestíveis; PB-d corresponde a proteína bruta digestível; AG-d significa ácidos graxos digestíveis; FDNn-d corresponde a FDN corrigida para nitrogênio digestível.

Para o cálculo de CNF-d utilizou-se a equação: $\mathrm{CNF}-\mathrm{d}=0,98 \times[100-(\% \mathrm{~PB}+\% \mathrm{EE}+\% \mathrm{FDNn}+\mathrm{MM})]$ $\mathrm{x}$ PAF; onde PAF é um fator de ajuste igual a 1 para todos os alimentos conforme NRC (2001). Para o cálculo da PB-d e AG-d usaram-se as equações: $\mathrm{PB}-\mathrm{d}=\mathrm{PB} \times \exp [-1,2 \times(\mathrm{PIDA} / \mathrm{PB})]$ e AG-d = EE -1 ; considerando que para alimentos com teores de $\mathrm{EE}<1, \mathrm{AG}-\mathrm{d}=0$. Para o cálculo de FDN-d utilizouse a expressão: FDNn-d =0,75 x (FDNn - Lig) x $\left[1-(\mathrm{Lig} / \mathrm{FDNn})^{0,667}\right]$; onde FDNn $=$ FDN - PIDN.

Perfis acumulativos de produção de gases in vitro foram obtidos utilizando a metodologia de Theodorou et al. (1994), modificada por Mauricio et al. (1999). Foram introduzidas $500 \mathrm{mg}$ de amostra em frascos de vidro de $150 \mathrm{ml}$, os quais receberam 40ml de solução tampão de McDougal (McDOUGAL, 1949), previamente reduzida com $\mathrm{CO} 2$ ( $\mathrm{pH}$ 6,9). Posteriormente, foram adicionados, em cada frasco, $10 \mathrm{ml}$ de inóculo oriundo de dois bovinos fistulados no rúmen, alimentados com silagem de sorgo ad libitum, sob aspersão de $\mathrm{CO}_{2}$ e mantidos a $39^{\circ} \mathrm{C}$ em incubadora. Para os ajustes de variação, foram incubados frascos considerados branco, contendo as soluções de incubação sem substrato. Os frascos foram hermeticamente fechados utilizando-se rolhas de borracha.

A partir deste momento, a pressão dos gases produzidos pela fermentação do substrato e acumulada nos frascos foi mensurada por meio de um manômetro, nos tempos de 1, 2, 3, 4, 5, 6, 9, $12,18,24,30,36,48,60,72,84,96$ e 144 horas, e convertida em volume, segundo recomendações de Mauricio et al. (1999).

Para estimativa dos parâmetros de cinética de fermentação ruminal de cada co-produto foi utilizado o modelo logístico bicompartimental, abaixo descrito, ajustado às curvas de produção cumulativa de gases (SCHOFIELD; PITT; PELL, 1994).

$\mathrm{V}(\mathrm{t})=\mathrm{Vf} 1 /(1+\exp \quad(2-4 * \mathrm{c} \quad *(\mathrm{~T}-\mathrm{L})))+\mathrm{Vf} 2 /$ $(1+\exp (2-4 * \mathrm{c} *(\mathrm{~T}-\mathrm{L})))$, onde:

Vf1= volume máximo de gás da fração de carboidratos não estruturais;

$\mathrm{Vf2}=$ volume máximo de gás da fração de carboidratos estruturais;

$\mathrm{C}=$ taxa de degradação $\left(\% \mathrm{~h}^{-1}\right)$;

$\mathrm{T}$ e $\mathrm{L}=$ tempos de incubação (horas) e a latência (horas).

Os dados foram submetidos à análise de variância e as medias comparadas pelo teste de Tukey a 5\% de probabilidade, utilizando o programa estatístico SAS (2001). Obrigatoriamente, todos os conjuntos de dados foram testados, com a intenção de assegurar que todas as premissas de análise de 
variância (aditividade do modelo, independência dos erros, normalidade dos dados e homocedasticidade) estivessem sendo respeitadas.

\section{Resultados e Discussão}

Pode-se observar pela composição bromatológica dos co-produtos (Tabela 1) que a torta de girassol e torta de crambe apresentaram os menores valores de proteína bruta (26,07 e $29,17 \%$, respectivamente). No entanto, estes foram os co-produtos, juntamente com a torta de soja, que apresentaram menores valores médios de proteína insolúvel em detergente ácido $(5,73,5,57$ e $5,06 \%$, respectivamente), o que pode indicar maior disponibilidade de proteína para os microrganismos ruminais e para digestibilidade intestinal da proteína (SNIFFEN et al., 1992).

Tabela 1. Composição química (\%) do farelo de crambe, farelo de algodão, torta de crambe, torta de soja e torta de girassol.

\begin{tabular}{lccccc}
\hline Item & $\begin{array}{c}\text { Farelo de } \\
\text { Crambe }\end{array}$ & $\begin{array}{c}\text { Farelo de } \\
\text { algodão }\end{array}$ & $\begin{array}{c}\text { Torta de } \\
\text { crambe }\end{array}$ & $\begin{array}{c}\text { Torta de } \\
\text { soja }\end{array}$ & $\begin{array}{c}\text { Torta de } \\
\text { girassol }\end{array}$ \\
\hline MS $(\%)$ & 89,76 & 91,80 & 93,33 & 90,83 & 92,91 \\
MO $^{1}$ & 93,19 & 94,87 & 95,86 & 95,15 & 95,53 \\
PB $^{1}$ & 37,07 & 44,13 & 29,17 & 41,27 & 26,07 \\
FDN $^{1}$ & 36,14 & 32,76 & 33,08 & 8,46 & 36,36 \\
FDNcp & 22,52 & 27,85 & 27,01 & 2,52 & 30,17 \\
DDA $^{1}$ & 27,79 & 20,95 & 24,75 & 6,28 & 27,23 \\
PIDN $^{2}$ & 12,84 & 4,36 & 4,04 & 5,93 & 5,61 \\
$\mathrm{PIDA}^{2}$ & 8,97 & 7,73 & 5,57 & 5,06 & 5,73 \\
$\mathrm{LIG}^{1}$ & 10,98 & 6,85 & 6,74 & 1,25 & 15,22 \\
$\mathrm{EE}^{1}$ & 3,40 & 1,40 & 24,90 & 24,79 & 22,02 \\
$\mathrm{MM}^{1}$ & 6,81 & 5,13 & 4,14 & 4,85 & 4,47 \\
$\mathrm{NDT}^{1}$ & 58,61 & 60,09 & 92,87 & 108,53 & 81,04 \\
$\mathrm{CT}^{1}$ & 52,71 & 49,34 & 41,79 & 29,09 & 47,45 \\
$\mathrm{CNF}^{1}$ & 30,20 & 21,49 & 14,78 & 26,57 & 17,28 \\
$\mathrm{CF}^{1}$ & 22,52 & 27,85 & 27,01 & 2,52 & 30,17 \\
\hline
\end{tabular}

${ }^{1}(\%$ na MS); $2(\% \mathrm{~PB})$; NDT, nutrientes digestíveis totais; $\mathrm{CF}$, carboidratos fibrosos.

Fonte: Elaboração dos autores.

O teor de PB do farelo de crambe encontrado neste trabalho $(37,07 \%)$ está próximo do valor (34,6\%) encontrado por Anderson et al. (1993).

Os valores de FDN das tortas de soja e crambe $(8,46$ e $33,08 \%)$ foram inferiores aos apresentados por Goes et al. (2010) (12,32 e 48,9\%). Já para a torta de girassol foram encontrados neste trabalho, valores de $36,36 \%$, os quais foram superiores aos relatados por Goes et al. (2010) (32,26\%). Segundo estes pesquisadores, estas variações demonstram a falta de padronização destes produtos, pois dependem da variedade plantada e do processamento do grão.

A presença de lignina tende a aumentar a fração indigerível, reduzindo, dessa forma, a fração potencialmente digerível. Os maiores teores de lignina na base seca foram encontrados na torta de girassol e no farelo de crambe $(15,22$ e 10,98\%, 
respectivamente), e o menor na torta de soja $(1,25 \%)$.

Quanto aos teores de carboidratos não fibrosos (CNF), farelo de crambe e torta de soja apresentaram-se como boas fontes $(30,20$ e 26,57\%, respectivamente). Os CNF são importantes constituintes químicos, pois representam fontes de rápida disponibilidade de energia para os microrganismos ruminais, sendo esta fração a que suporta o crescimento inicial de microorganismos.

Após 144 horas de incubação, a torta de soja foi o co-produto com o maior volume final da produção cumulativa de gás $\left(162,93 \mathrm{~mL} \mathrm{~g}^{-1}\right.$ de MS incubada) e a torta de girassol apresentou o menor volume com 96,97 mL. As médias para o farelo de algodão, farelo de crambe e torta de crambe foram, respectivamente, de 156,11, 154,64 e 122,69 mL (Figura 1).

Assumindo-se que o tempo médio de retenção do alimento no rúmen é de 48 horas, quanto maior for a degradação até este tempo, melhor é a qualidade fermentativa do alimento. É possível visualizar na Figura 1 que a torta de girassol e o farelo de algodão foram os co-produtos com menor acúmulo de gás ate 48 horas, em termos proporcionais, sendo que 82,02 e $79,52 \%$ do volume final de gás acumulado foi produzido até este tempo respectivamente, para os dois alimentos, enquanto que para a torta de soja, torta de crambe e farelo de crambe, esses percentuais foram de 95,7, 96,42 e 93,85\%, respectivamente.

Figura 1. Produção total de gás em função dos tempos de incubação.

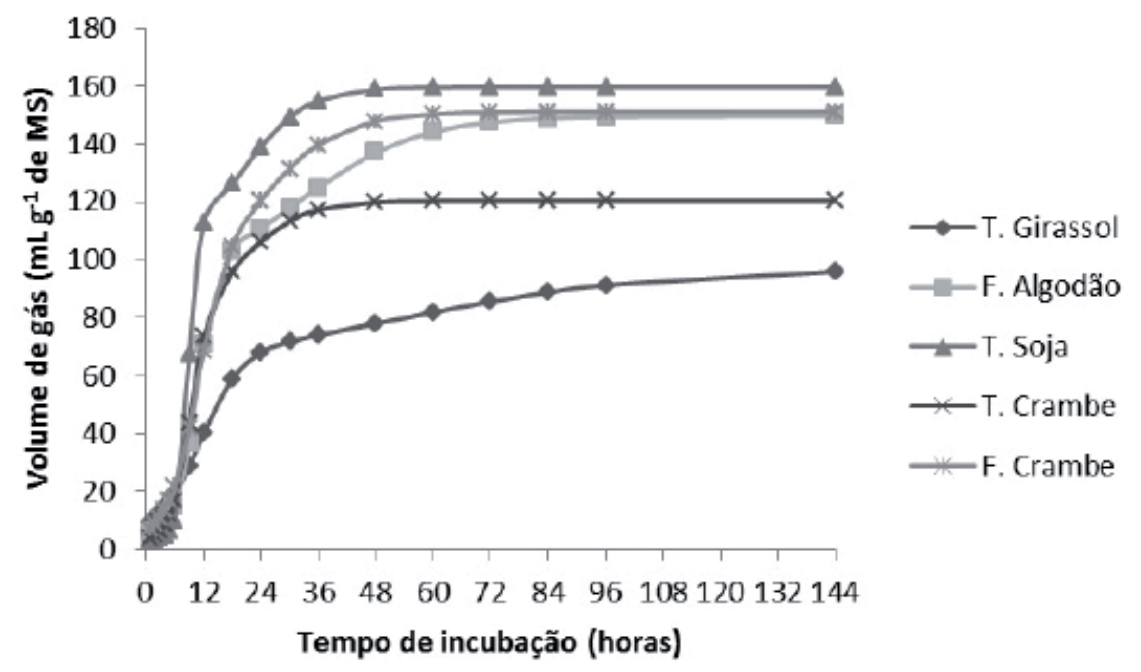

Fonte: Elaboração dos autores.

Pode-se verificar que houve variação significativa para os parâmetros cinéticos de degradação in vitro dos co-produtos em estudo (Tabela 2). Esta variação confere diferenças importantes entre esses co-produtos, uma vez que resulta em maior ou menor digestibilidade dos mesmos.

A torta de soja apresentou maior taxa de degradação (kdCNF) de CNF ( $\mathrm{P}<0,05 \%)$, quando comparada aos demais alimentos, resultando em maior volume final de gases (VfCNF). Este fato está associado ao reduzido teor de CF (2,52\%) neste alimento, e portanto, o alto VfCNF é consequência da elevada taxa de degradação de CNF. 
Tabela 2. Médias de volume final de produção de gases oriundos da degradação de carboidratos fibrosos (VfCF) e não-fibrosos (VfCNF), suas respectivas taxas de degradação (KdCF e KdCNF) e lag time da torta de girassol, farelo de algodão, torta de soja, torta de crambe e farelo de crambe.

\begin{tabular}{lccccccc}
\hline ITEM & \multicolumn{5}{c}{ Tratamentos } & Valor P & \multirow{2}{*}{ EPM } \\
\cline { 2 - 6 } & T. Girassol & F. Algodão & T. Soja & T. Crambe & F. Crambe & Trat & \\
\hline $\mathrm{VfCNF}^{1}$ & $63,28 \mathrm{~d}$ & $89,83 \mathrm{ab}$ & $102,07 \mathrm{a}$ & $75,03 \mathrm{~cd}$ & $84,75 \mathrm{bc}$ & 0,0002 & 4,37 \\
$\mathrm{KdCNF}^{2}$ & $0,0575 \mathrm{~d}$ & $0,1271 \mathrm{bc}$ & $0,2884 \mathrm{a}$ & $0,1350 \mathrm{~b}$ & $0,0935 \mathrm{~cd}$ & 0,0001 & 0,01 \\
lag time $^{3}$ & $2,49 \mathrm{~d}$ & $6,63 \mathrm{ab}$ & $7,01 \mathrm{a}$ & $5,50 \mathrm{bc}$ & $5,20 \mathrm{c}$ & 0,0001 & 0,44 \\
$\mathrm{VfCF}^{1}$ & $33,70 \mathrm{~d}$ & $60,03 \mathrm{~b}$ & $57,67 \mathrm{~b}$ & $45,37 \mathrm{c}$ & $66,44 \mathrm{a}$ & 0,0001 & 1,99 \\
$\mathrm{KdCF}^{2}$ & $0,0095 \mathrm{~d}$ & $0,0199 \mathrm{c}$ & $0,0380 \mathrm{a}$ & $0,0376 \mathrm{a}$ & $0,0289 \mathrm{~b}$ & 0,0001 & 0,001 \\
\hline $\mathrm{r}^{2}$ & 0,99 & 0,99 & 0,99 & 0,99 & 0,99 & - & - \\
\hline
\end{tabular}

${ }^{1}(\mathrm{~mL}) ;{ }^{2}(\% \mathrm{~h}-1) ;{ }^{3}$ (horas); EPM, erro padrão da média.

Médias seguidas de letras iguais, nas linhas, não diferem estatisticamente pelo teste Tukey $(\mathrm{P}<0,05)$.

Fonte: Elaboração dos autores.

O farelo de algodão e o farelo de crambe apresentaram taxa de degradação de CNF e produção de gases similares $(\mathrm{P}<0,05)$, o que permite inferir que estes alimentos apresentam o mesmo comportamento quanto à fermentação ruminal de CNF. Apesar da torta de crambe não diferir $(\mathrm{P}>0,05)$ do farelo de crambe quanto a produção de gás (VfCNF) na degradação de $\mathrm{CNF}$, houve diferença na taxa de degradação (kdCNF), sendo o maior valor verificado para a torta. Isto pode estar associado à temperatura utilizada na produção do farelo, o que pode interferir na taxa de degradação.

O tempo de colonização (lag time) é um parâmetro importante e está relacionado com a degradação da fração fibrosa (MERTENS; LOFTEN, 1980). O maior tempo de colonização foi requerido para a torta de soja e o menor para a torta de girassol.

Quanto 'as taxas de degradação dos carboidratos fibrosos, houve diferença significativa entre os alimentos $(\mathrm{P}<0,05)$ (Tabela 2). Observa-se que as taxas de degradação de CF foram de 0,0095; 0,0199; 0,0380; 0,0376 e 0,0289 , respectivamente, para torta de girassol, farelo de algodão, torta de soja, torta de crambe e farelo de crambe. Não foram encontrados na literatura, taxas de degradação de carboidratos fibrosos de alimentos semelhantes aos utilizados neste trabalho, e avaliados pela técnica de produção de gás, dificultando, portanto, a comparação dos mesmos. Foram encontrados, vários trabalhos, avaliando alimentos volumosos. Neste sentido, pode-se verificar que os valores encontrados neste trabalho foram relativamente baixos quando comparados com os encontrados por Cabral (2002), que trabalharam com capim elefante, capim tifton, silagem de milho, silagem de sorgo, feno de alfafa e feno de coastross, encontrando valores de 0,$055 ; 0,049$; 0,$036 ; 0,045$ e 0,040 .

Observa-se que a maior taxa de degradação de carboidratos fibrosos foi observado com a torta de soja e torta de crambe $(\mathrm{P}<0,05)$ (Tabela 2). Esses alimentos apresentaram menor concentração de lignina (Tabela 1) facilitando o acesso dos microorganismos aos carboidratos fibrosos. Os carboidratos fibrosos compreendem as fibras disponíveis e indisponíveis, representados pela celulose e hemicelulose, juntamente com a lignina, sendo parcialmente disponíveis no rúmen (MERTENS, 1997). Essa maior taxa de degradação sugere que a torta de soja e a torta de crambe causam menor efeito de repleção ruminal, quando comparada aos outros alimentos estudados. O contrário pode ser observado para a torta de girassol que apresentou a menor taxa de degradação, podendo provocar um grande efeito de repleção ruminal, limitando o consumo de matéria seca e prejudicando a produção animal que exigem uma grande demanda nutricional.

A maior produção de gás oriunda da degradação de CF foi obtida no farelo de crambe, podendo-se sugerir parcialmente que esta fração apresenta boa fermentação ruminal em função do equilíbrio encontrado entre energia e compostos nitrogenados fornecidos aos 
microrganismos, melhorando-se a degradação da fração fibrosa. Porém, segundo Getachew et al. (2004), a quantidade de gases produzidos de um alimento em incubação reflete a produção de ácidos graxos de cadeia curta, que são a principal fonte de energia dos ruminantes. A produção de gás surge da degradação microbiana dos alimentos e indiretamente da reação do tampão com os ácidos gerados como resultado da fermentação. Espera-se que um alimento que possui grande parte dos seus carboidratos na forma de amido, presente nos grãos, produza mais propionato do que acetato. Segundo Beuvink e Spoelstra (1992), a produção de propionato gera somente $0,87 \mathrm{~mol} / \mathrm{mol}$ de $\mathrm{AGV}$ por meio da produção indireta e nenhum gás diretamente, enquanto que, a produção de acetato gera 2 moles de $\mathrm{CO} 2$ por mol de glicose pela produção direta, e $0,87 \mathrm{~mol} / \mathrm{mol}$ de AGV, indiretamente. Assim, os autores sugeriram que a comparação de alimentos somente com base na produção acumulativa de gás pode levar a conclusões equivocadas sobre o valor energético dos alimentos, uma vez que a proporção molar dos AGVs afeta o volume de gás produzido por mol de hexose fermentada.

Por outro lado, observou-se menor produção de gás (VfCNF) na degradação de CF da torta de girassol que pode estar associado ao alto teor de lignina (Tabela 1). Esta estrutura está diretamente correlacionada à digestibilidade do alimento, e neste caso, provavelmente os microorganismos colonizaram as partículas de alimento, mas esbarraram em uma estrutura bastante lignificada, reduzindo a taxa de degradação e conseqüentemente a produção de gás.

As diferenças na produção de gás decorrentes das degradações de CNF e CF podem ser observadas para cada co-produto individualmente na Figura 2.

Figura 2. Produção de gás oriunda da degradação dos CNF, dos $\mathrm{CF}$, e volume total, em função do tempo de incubação, de: a) torta de girassol; b) torta de crambe; c) farelo de algodão; d) farelo de crambe; e) torta de soja.

\section{a}
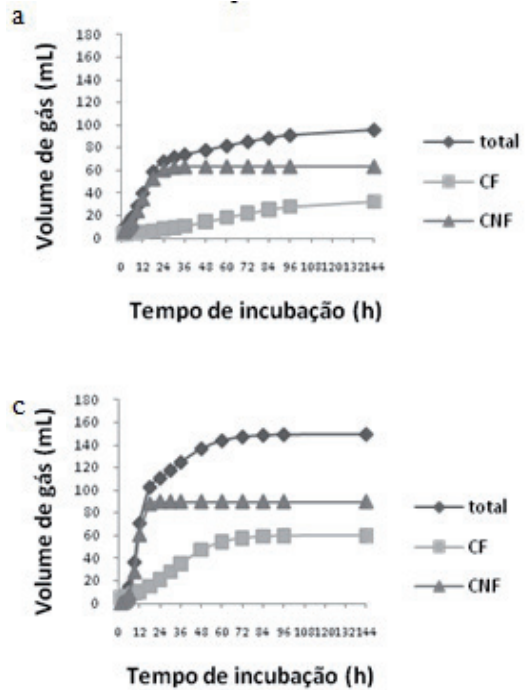

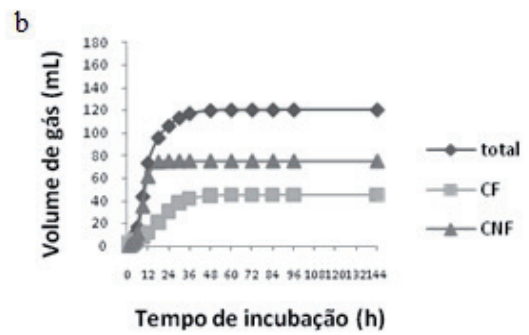

d

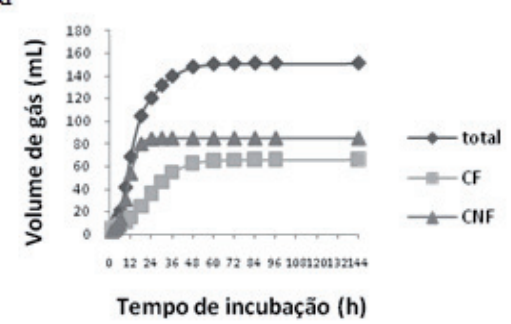

Fonte: Elaboração dos autores.

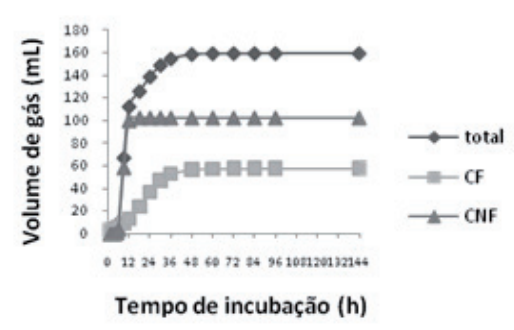




\section{Conclusões}

A torta de soja e a torta de crambe são co-produtos com um bom perfil de cinética de fermentação ruminal no que diz respeito à degradação de carboidratos $\neg$ não fibrosos e carboidratos fibrosos, sendo potenciais fornecedores de energia na dieta de ruminantes.

Os elevados teores de lignina provavelmente influenciam negativamente a degradação de carboidratos não fibrosos e carboidratos fibrosos da torta de girassol.

\section{Referências}

ABDAlla, A. L.; SILVA FILHO, J. C.; GODÓI, A. R.; CARMO, C. A.; EDUARDO, J. L. P. Utilização de subprodutos da indústria de biodiesel na alimentação de ruminantes. Revista Brasileira de Zootecnia, Viçosa, MG, v. 37, p. 260-258, 2008. Especial.

AGÊNCIA NACIONAL DO PETRÓLEO, GÁS NATURAL E BIOCOMBUSTÍVEIS - ANP. Boletim Mensal do Biodiesel, Outubro de 2010. Brasília, DF, 2010.

ANDERSON, V. L.; SLANGER, W. D.; BOYLES, S. L.; BERG, P. T. Crambe meal is equivalent to soybean meal for backgrounding and finishing beef steers. Journal of Animal Science, Champaign, v. 71, n. 10, p. 2608-2613, 1993.

BEUVINK, J. M. W.; SPOELSTRA, S. F. Interactions between substrate, fermentation end-products, buffering systems and gas production upon fermentation of different carbohydrates by mixed rumen microorganisms in vitro. Applied Microbiology and Biotechnology, New York, v. 37, n. 4, p. 505-509, 1992.

CABRAL, L. S. Avaliação de alimentos para ruminantes por intermédio de métodos in vivo e in vitro. 2002. Tese (Doutorado em Zootecnia) - Universidade Federal de Viçosa, Viçosa, MG.

CONFEDERAÇÃO NACIONAL DA AGRICULTURA - CNA. Composição da alimentação de confinamentos em Goiás, Mato Grosso e São Paulo. Ativos da Pecuária de Corte, ano 2, ed. 17. Brasília, DF, junho de 2010.

GETACHEW, G.; ROBINSON, P. H.; DEPETERS, E. J.; TAYLOR, S. J. Relationship between chemical composition, dry matter degradation and in vitro gas production of several ruminant feeds. Animal Feed Science and Technology, Amsterdam, v. 111, n. 1-4, p.
57-71, 2004.

GOES, R.; SOUZA, K.; PATUSSI, R.; CORNELIO, T.; OLIVEIRA, E.; BRABES, K. Degradabilidade in situ dos grãos de crambe, girassol e soja, e de seus coprodutos em ovinos. Acta Scientiarum. Animal Sciences, Maringá, v. 32, n. 3, p. 271-277, 2010.

MAURICIO, R. M.; MOULD, F. L.; DHANOA, M. S.; OWEN, E.; CHANNA, K. S.; THEODOROU, M. K. A semi-automated in vitro gas production technique for ruminants feedstuff evaluation. Animal Feed Science and Technology, Amsterdam, v. 79, n. 4, p. 321-330, 1999.

McDOUGAL, E. I. Studies on ruminal saliva. 1. The composition and output of sheep's saliva. Biochemical Journal, London, v. 43, n. 1, p. 99-109, 1949.

MERTENS, D. R. Creating a system for meeting the fiber requirements of dairy cows. Journal of Dairy Science, Savoy, v. 80, n. 7, p. 1463-1481, 1997.

MERTENS, D. R.; LOFTEN, J. R. The effect of starch on forage fiber digestion kinetics in vitro. Journal of Dairy Science, Savoy, v. 63, n. 9, p. 1437-1446, 1980.

MIZUBUTI, I. Y.; PINTO, A. P.; RAMOS, B. M. O.; PEREIRA, E. S. Métodos laboratoriais de avaliação de alimentos para animais. Londrina: EDUEL, 2009. 228 p.

NATIONAL RESEARCH COUNCIL - NRC. Nutrient requirements of dairy cattle. $7^{\text {th }}$ ed. Washington. DC: National Academy Press. 2001. 381 p.

SCHOFIELD, P.; PITT, R. E.; PELL, A. N. Kinetic of fiber digestion from in vitro gas production. Journal of Animal Science, Champaign, v. 72, n. 11, p. 2980-2991, 1994.

SNIFFEN, C. J.; O'CONNOR, J. D.; VAN SOEST, P. J.; FOX, D. G.; RUSSEL, J. B. A net carbohydrate and protein system for evaluating cattle diets. II Carbohydrate and protein availability. Journal of Animal Science, Champaign, v. 70, n. 11, p. 3562-3577, 1992.

STATISTICAL ANALYSIS SYSTEMS - SAS. User's guide. Version 8. 1.ed. Cary: SAS Institute, 2001. 956 p.

THEODOROU, M. K.; WILLIAMS, B. A.; DHANOA, M. S.; McALlAN, A. B.; FRANCE, J. A simple gas production method using a pressure transducer to determine the fermentation kinetic of ruminant feeds. Animal Feed Science and Technology, Amsterdam, v. 48, n. 3, p. 185-197, 1994. 